\title{
Prácticas emergentes en actividad física y deporte
}

\author{
Lina M. Cardona ${ }^{1}$, Fernando Avendaño², y Juan P. Marín ${ }^{3}$ \\ (1) Politécnico Colombiano Jaime Isaza Cadavid, Facultad de Educación Física, Recreación y Deporte. Medellín, \\ Colombia. (Correo-e: linacardona@elpoli.edu.co) \\ (2) Universidad Nacional de Rosario, Facultad de Humanidades y Artes. Rosario, Argentina. \\ (Correo-e: fernandoavendano90@gmail.com) \\ (3) Politécnico Colombiano Jaime Isaza Cadavid, Facultad de Educación Física, Recreación y Deporte. Medellín, \\ Colombia. (Correo-e: juanmarin@elpoli.edu.co)
}

Recibido Dic. 4, 2020; Aceptado Feb. 1, 2021; Versión final Mar. 2, 2021, Publicado Jun. 2021

\begin{abstract}
Resumen
El objetivo de este estudio fue determinar las prácticas emergentes en actividad física y deporte que evolucionan en Medellín (Colombia) y su relación con el currículum de un programa profesional académico en deporte. El enfoque metodológico fue mixto, en la fase cuantitativa se aplicó un cuestionario a 428 sujetos y en la fase cualitativa una entrevista semiestructurada a un grupo focal de personas vinculadas con actividad física y deporte. Las prácticas emergentes con mayor evolución en el contexto local son en su orden: entrenamiento funcional, deportes adaptados, CrossFit, Ultimate y ciclo montañismo. Por su parte el grupo focal considera que las cinco prácticas emergentes con mayor evolución son: skateboarding, street workout, parkour, entrenamiento funcional y CrossFit. Con base en los resultados, se propone un plan de actualización del currículo, pertinente con las actividades prácticas emergentes, que sin pretender ser excluyente, ni totalitario, incluya espacios formativos alternativos en la actividad física y el deporte.
\end{abstract}

Palabras clave: prácticas emergentes; actividad física; deporte; educación superior; currículo

\section{Emerging practices in physical activity and sports}

\begin{abstract}
The main objective of the present study was to determine the emerging practices in physical activity and sports that are evolving in Medellín (Colombia) and their relationships with the academic curriculum of a sports degree program. The methodological approach was mixed. In the quantitative phase, a questionnaire was applied to 428 people. In the qualitative phase, a semi-structured interview was conducted in a focus group with people linked to physical activities and sports. The results showed that the most prevalent emerging practices were: functional training, adapted sport, CrossFit, Ultimate, and mountain biking. The focus group considered that the five emerging practices with most growth were: skateboarding, street workout, parkour, functional training, and CrossFit. Based on the results obtained, an updated plan is proposed for an academic sports degree curriculum including relevant emerging practices and activities that without pretending to be exclusive or radical, includes alternative training spaces for physical activities and sports.
\end{abstract}

Keywords: emerging practices; physical activity; sport; higher education; curriculum 


\section{INTRODUCCIÓN}

En los últimos años, se ha venido presentando un auge de las denominadas prácticas emergentes (PE) o nuevas tendencias en la Actividad Física y el Deporte, que en la actualidad congregan a una creciente comunidad de jóvenes practicantes. Se habla de tendencia porque implica un cambio en la forma como las personas se están comportando. El tema de nuevas tendencias es un concepto polisémico, de ahí que en el análisis de la información encontrada, se encuentren diferentes acepciones al respecto, así por ejemplo, se habla de tendencias consolidadas o emergentes en el sector del fitness (Veiga et al., 2017); nuevas tendencias emergentes (Thompson, 2019); prácticas corporales no convencionales y deportes alternativos emergentes (Zaragoza, 2017); prácticas deportivas emergentes, deportes extremos, de aventura y nuevas tendencias (Gaviria et al., 2015); prácticas emergentes (Águila y Sicilia, 2014)

En la sociedad se están produciendo cambios, sociales, éticos y culturales, que afectan la educación física y el deporte, y en este contexto las nuevas tendencias que hoy se encuentran, invitan a reflexionar sobre un proceso disruptivo que se ha ido consolidando como un fenómeno que viene propiciando una renovación radical, apalancado en las tecnologías de la información y la comunicación, herramientas que le han permitido responder al reconocimiento del pluralismo y la diversidad global (Kumar, 2017). A pesar del despliegue de los medios de comunicación por los deportes y actividad física convencional o deportes competitivos tradicionales, es claro que ha habido un cambio en los patrones de participación de las personas en actividades de educación física y el deporte, y por tanto, estos cambios deben verse como oportunidades para que las instituciones de Educación Superior que forman profesionales en la actividad física y el deporte, adecuen el currículo de los programas basados en una comprensión del actual ecosistema de la educación física y el deporte, es decir, un plan de estudios más inclusivo que equilibre la competición y el deporte con otras dimensiones menos competitivas en educación física (OECD, 2019, p.54). Por lo tanto, no se puede seguir formando profesionales en actividad física y deporte fundamentados exclusivamente en el slogan olímpico el más fuerte, más alto, más rápido (Garganta, 2009)

Partiendo de la interpretación de Inglehart, al sistema deportivo, García y Llopis (2011) diferencian los comportamientos deportivos de orientación moderna de los de orientación posmoderna, al respecto expresan que los primeros han impulsado de manera preponderante el deporte reglamentado por las federaciones deportivas, y los segundos invitan a practicar y vivir el deporte de una forma diferente, es decir, dirigido hacia la recreación y el ocio y no tanto a la competencia reglamentada, buscando obtener nuevas experiencias individuales y grupales.

Estas últimas actividades implican un culto al riesgo, al contacto directo y cuidado del entorno natural y la transgresión de reglas previamente institucionalizadas, que permiten la exploración deportiva en nuevos ambientes en los que no están presentes las características del deporte clásico o tradicional; en esta perspectiva, se considera que con ello se generan espacios de construcción deportiva de manera lúdica, de riesgo y en contacto con la naturaleza circundante (Mata, 2002). Con base en este criterio y en oposición a las prácticas convencionales, las prácticas emergentes se configuran como un fenómeno complejo alterno, que fomenta el espíritu de aventura más que el de competitividad y en algunos casos, sin pretender una reglamentación estandarizada, pues se basa en la nobleza del espíritu deportivo, además, sus espacios físicos y lugares de práctica por lo general son marginales, "casi siempre son manifestaciones postmodernas y en ocasiones son expresiones contraculturales" (Gaviria et al., 2015). En general se puede decir que las prácticas emergentes o nuevas tendencias, se realizan en lugares abiertos privilegiando el uso de espacios públicos, parques, plazoletas y vías o también en espacios naturales. Según Gaviria et al. (2015) las prácticas deportivas emergentes incluyen nuevas tendencias deportivas y modalidades reconocidas como deporte por su tradición e historia, algunas de ellas federadas, como es el caso del motociclismo y el parapentismo.

Por el contrario hay algunas de estas prácticas que están lejos de constituir sus ligas y federaciones, pues tienen un carácter emergente en el que persiste el desinterés por participar de las tendencias que se dan en las actividades físicas tradicionales. Gaviria et al. (2015) ponen como ejemplo la práctica del skate y dicen que "este tipo de disciplina puede incluir tanto prácticas extremas urbanas o de aventura, como prácticas que no implican un riesgo prevalente", tal es el caso del frisbee, freestyle, agility y salto doble cuerda. López (2011) señala que son los jóvenes quienes, de forma individual y colectiva, vienen participando activamente en la construcción del contexto social y en la generación de prácticas alternativas, reforzando la idea de que el deporte y las actividades físicas son un fenómeno de naturaleza esencialmente social, expuestas a las incesantes y veloces transformaciones que se están produciendo en el seno de la sociedad contemporánea.

El deporte, en un sentido amplio abarca toda la práctica física y puede ser considerado un fenómeno social que progresa al igual que el desarrollo de las sociedades modernas y considera un reflejo sociocultural de la situación posmoderna de la época actual”. Según Mata (2002) el deporte constituye asimismo un factor de etnicidad, al crear una identidad subcultural propia, marginal y en esencia, contracultural al sistema; la misma concepción de deporte alternativo y su uso como componente de evasión del individuo han establecido un 
abordaje contracultural en relación al mismo, pues exhibe patrones de actuación, señales de identidad y valores esencialmente diferentes a los que proclama y revela la concepción clásica de deporte.

En el ámbito Nacional, las prácticas emergentes vienen presionando con fuerza el ecosistema social del deporte, promoviendo la creación de políticas, infraestructura, nuevo instrumental y equipamiento, capacitación y formación de capital humano, eventos deportivos y recreativos, redes de conocimiento, apropiación de nuevos espacios, constituyéndose en una nueva economía, soportada por un sector productivo y empresarial, que viene respondiendo con pertinencia a estas nuevas dinámicas. Sánchez (2001) plantea que "el proceso de transformación de la sociedad tiene su reflejo en el deporte, que irá modificando su naturaleza y sus formas para adaptarse y dar respuesta a las expectativas generadas por el surgir de nuevos valores sociales".

En el contexto de la ciudad de Medellín-Colombia, la mayoría de estas prácticas o tendencias son foráneas, no obstante, han logrado evolucionar con independencia y permear de manera significativa espacios sociales y educativos, gracias a la globalización, a los sistemas de información y comunicación, a las redes sociales y a los entornos digitales, sin embargo, pese al auge, diversificación y aceptación que han adquirido en el mundo deportivo y la actividad física, en el contexto de la educación superior, aun no logran permear el currículo de los programas de formación en deporte, dado que, se siguen seleccionado contenidos y actividades prácticas que continúan priorizando los deportes y actividades físicas convencionales. Rath (2017) plantea que los deportes competitivos fueron priorizados en los planes de estudio de educación física en las escuelas y universidades. Ahora bien, esto no quiere decir que, la atención a los patrones de cambio de comportamiento, por las nuevas tendencias o disciplinas alternativas no convencionales en la educación física o el deporte, desplacen a la formación en disciplinas convencionales, sino que, los programas de educación superior generen espacios curriculares para la enseñanza de estas nuevas disciplinas, integrando objetivos, contenidos y actividades pedagógicas y didácticas, que permitan responder con pertinencia a estas nuevas dinámicas y alcanzar aprendizajes significativos.

Para el caso del programa de profesional en deporte, un análisis detallado de los documentos institucionales y del plan de estudio, indica que, hasta ahora, la comunidad educativa adscrita al programa, no ha manifestado un interés colectivo por incorporar al currículo de forma explícita las prácticas emergentes, pese a que, los informes de seguimiento a graduados del programa indican que, en este campo, existe un dinámico medio laboral en el cual estos se viene desempeñando. El actual plan de estudio sigue privilegiando contenidos y prácticas convencionales, que ejercen una posición dominante en el currículum, haciéndolo poco pertinente con la pluralidad, alteridad, interdisciplinariedad y espontaneidad implícita en el concepto de práctica emergente construida socialmente por los jóvenes.

En este sentido, el objetivo de la investigación realizada fue determinar las prácticas emergentes en actividad física y deporte que evolucionan en la ciudad de Medellín y su relación con el currículum del Programa Profesional en Deporte de la Facultad de Educación Física del Politécnico Colombiano Jaime Isaza Cadavid. Desde las inquietudes y problemas que surgen para la elaboración de este trabajo, se decide iniciar la etapa exploratoria en el campo de la actividad física, con el análisis de estudios relacionados con las áreas del aprendizaje, la actividad física escolar, la promoción de Salud en la escuela y las relaciones entre actividad física y currículo.

\section{METODOLOGÍA}

El tipo de investigación fue mixto, no experimental descriptiva y se tiene en cuenta la propuesta de Polit y Hungler (2005) de corte transversal dado que no hubo manipulación de variables independientes y tuvo la intención de caracterizar las prácticas emergentes y su pertinencia en el currículo del programa profesional en deporte del Politécnico Colombiano Jaime Isaza Cadavid. En la fase cuantitativa se empleó un diseño descriptivo y en la fase cualitativa se llevó a cabo un análisis documental del programa y ocho (8) entrevistas.

\section{Instrumentos para la recolección de datos cuantitativos}

Se basaron en la aplicación de una encuesta tipo cuestionario. Para tal efecto se diseñaron cuatro cuestionarios estructurados, dirigidos a 67 docentes, 119 graduados, 56 empresarios y 186 estudiantes, autoaplicados, tipo likert de respuesta graduada (1 a 5) puntuaciones y de selección. El Procesamiento de datos y análisis estadístico cuantitativo, fue digitada en una base de datos en Excel (2014) y procesada en el software SPSS v24. La muestra para la recolección de información se elaboró a partir de un muestreo probabilístico estratificado (Grisales, 2000), teniendo en cuenta criterios de confiabilidad (95\%), error (5\%), probabilidad de ocurrencia $(P=50 \%)$, probabilidad de no ocurrencia $(\mathrm{Q}=50 \%)$, población $(\mathrm{N}=777)$ y muestra final $(n=428)$. La forma en que escogió la muestra se presenta en la tabla 1. La selección de los sujetos se realizó a partir de un procedimiento aleatorio simple. Para la elección de los grupos y los participantes se empleó una tabla de números aleatorios (Polit y Hungler, 2005). 
Tabla 1. Valores calculados de la muestra para cada uno de los grupos de interés

\begin{tabular}{|l|c|c|c|c|c|}
\hline \multicolumn{1}{|c|}{ Grupo de interés } & Estudiantes & Docentes & Empresarios & Egresados & Total \\
\hline Población (N) & 204 & 100 & 80 & 393 & 777 \\
\hline Muestra (n) & 100 & 66 & 56 & 131 & 353 \\
\hline Muestra ajustada (ñ) & 67 & 40 & 33 & 98 & 238 \\
\hline Muestra lograda & 186 & 67 & 56 & 119 & 428 \\
\hline
\end{tabular}

\section{Pruebas de validación de cuestionarios y consistencia entre jueces}

Como se observa en la tabla 1, se aplicaron en total 428 cuestionarios, que recogen distintos aspectos a partir de los cuales se acerca a la valoración de las Prácticas Emergentes, en el contexto de la formación del Profesional en Deporte del PCJIC. La construcción de los ítems se hizo a partir de una revisión bibliográfica en bases de datos especializadas y textos claves de la temática; y de acuerdo con esta información se construyeron los 29 ítems que se sometieron a la validación de contenido, mediante el juicio de diez expertos, los cuales son docentes universitarios activos, con experiencia en docencia, investigación y cargos directivos relacionados con el deporte y con título mínimo de maestría. La confiabilidad fue determinada por medio del método de consistencia interna a través del coeficiente alfa de Cronbach. Como ya se anotó el juicio de expertos estaba relacionada con dos temas fundamentales: primero, si consideraban o no confiable considerar como prácticas emergentes, los deportes o actividades físicas incluidas en los 29 ítems y segundo, las prácticas emergentes que ellos considerarían incluir en el plan de estudios de un profesional en deporte.

Luego del juicio de expertos, para el primer caso, los cuestionarios pasaron de 29 a 25 items (ver tabla 2) y para el segundo caso pasaron de 29 a 15 ítems (ver tabla 4). Esta reducción se presenta porque se excluyeron los ítems cuyo valor CVR (Content Validity Ratio) o Índice de Validez de Contenido fue inferior a 0,5823. En promedio los cuestionarios presentaron valores $C V R=0,90$ para las Prácticas Emergentes, y de 0,74 para la inclusión de las prácticas emergentes en el plan de estudios. Luego haber obtenido resultados definitivos de la valoración por parte de los expertos, se procedió a aplicar una prueba piloto a 31 estudiantes (16\%), 12 docentes (18\%), 10 empresarios (18\%) y 40 graduados $(34 \%)$.

\section{Instrumentos para la recolección de datos cualitativos}

Las técnicas empleadas para la recolección de datos fueron el análisis documental relacionado con el programa de Profesional en Deporte y una entrevista semi-estructurada, dirigida a un grupo focal conformado por ocho personas vinculadas con la actividad física y el deporte representativos de la ciudad. En general los temas de la entrevista giraron en torno a la relación de las Prácticas Emergentes en la Actividad Física y deporte y su relación con el currículum del Programa Profesional en Deporte del Politécnico Colombiano Jaime Isaza Cadavid. Las preguntas que orientaron la entrevista fueron las siguientes: ¿Considera que las prácticas emergentes deben ser incluidas en el Currículo del Programa Profesional en Deporte?, ¿Cuáles prácticas emergentes conoce?, ¿Cuál práctica emergente practica?, ¿Cómo define las prácticas emergentes?

\section{RESULTADOS Y DISCUSIÓN}

Se presentan a continuación los resultados del estudio. Inicialmente se exponen los elementos relacionados con los datos cuantitativos y posteriormente se exhiben los datos cualitativos del grupo focal.

\section{Datos cuantitativos}

Una vez validado el instrumento, se procedió a aplicar el cuestionario a los cuatro actores del currículo: estudiantes, docentes, graduados y empresarios. El consolidado del análisis general de las respuestas, respecto a lo que la comunidad académica del Politécnico Colombiano considera prácticas emergentes en el contexto de la ciudad de Medellín (ver tabla 2 ) indica que, las cinco prácticas con mayores valores promedio fueron en su orden el Crossfit $(4,36)$, entrenamiento funcional $(4,35)$, deportes adaptados $(4,14)$, Street Workout $(4,11)$ y entrenamiento en suspensión $(4,11)$. Así mismo la de menor valor promedio fue agility con (3.73)

En el análisis de las prácticas emergentes teniendo en cuenta la muestra indagada, el Crossfit es la práctica emergente con mejor valor promedio en la población estudiantil $(4,36)$ y docente $(4,36)$; para el caso de los empresarios $(4,34)$ y los egresados fue el Entrenamiento Funcional $(4,51)$ (Ver tabla 3$)$ 
Tabla 2. Estadísticos descriptivos de Prácticas Emergentes

\begin{tabular}{|l|c|c|}
\hline Variables & Media & Desv. típ. \\
\hline Crossfit (metodología) & 4,36 & 0,88 \\
\hline Entrenamiento funcional & 4,35 & 0,85 \\
\hline Deportes adaptados & 4,14 & 1 \\
\hline Street Workout & 4,11 & 0,96 \\
\hline Entrenamiento en suspensión & 4,11 & 0,98 \\
\hline Deportes electrónicos & 3,74 & 1,21 \\
\hline Ultímate & 3,99 & 1,15 \\
\hline Rugby subacuático & 4,05 & 1,09 \\
\hline Kayak & 3,85 & 1,16 \\
\hline Raftin & 3,79 & 1,15 \\
\hline Agility & 3,73 & 1,19 \\
\hline Lacrosse & 3,84 & 1,15 \\
\hline Roller derby & 3,89 & 1,08 \\
\hline Slackline & 3,85 & 1,08 \\
\hline Salto doble cuerda & 3,81 & 1,04 \\
\hline Skateboarding & 3,88 & 1,08 \\
\hline Downhill & 3,94 & 1,09 \\
\hline Escalada & 3,99 & 1,07 \\
\hline Senderismo & 3,95 & 1,11 \\
\hline Orientación & 3,81 & 1,14 \\
\hline Caminata campo traviesa & 3,88 & 1,08 \\
\hline Ciclomontañismo & 3,91 & 1,15 \\
\hline Freestyle & 3,91 & 1,14 \\
\hline Parkour & 4,01 & 1,08 \\
\hline BMX & 4,11 & 1,12 \\
\hline n=428 & & \\
\hline
\end{tabular}

Tabla 3. Estadísticos descriptivos de Prácticas Emergentes de acuerdo a la muestra

\begin{tabular}{|c|c|c|c|c|c|c|c|c|}
\hline \multirow[b]{2}{*}{ Variables } & \multirow{2}{*}{$\begin{array}{c}\begin{array}{c}\text { Estudiantes } \\
(n=186)\end{array} \\
\text { Media }\end{array}$} & \multirow[b]{2}{*}{$\begin{array}{c}\text { Desv. } \\
\text { típ. }\end{array}$} & \multicolumn{2}{|c|}{ Docentes $(n=67)$} & \multicolumn{2}{|c|}{ Empresarios $(n=56)$} & \multicolumn{2}{|c|}{ Egresados $(n=119)$} \\
\hline & & & Media & $\begin{array}{c}\text { Desv. } \\
\text { típ. }\end{array}$ & Media & Desv. típ. & Media & Desv. típ. \\
\hline Crossfit (metodología) & 4,36 & 0,85 & 4,36 & 0,81 & 4,21 & 1,04 & 4,43 & 0,89 \\
\hline Entrenamiento funcional & 4,32 & 0,81 & 4,15 & 0,97 & 4,34 & 0,90 & 4,51 & 0,79 \\
\hline Deportes adaptados & 4,28 & 0,92 & 3,84 & 1,16 & 3,61 & 1,09 & 4,34 & 0,83 \\
\hline Street Workout & 4,06 & 0,96 & 4,22 & 0,93 & 4,16 & 0,91 & 4,11 & 0,99 \\
\hline Entrenamiento en suspensión & 4,09 & 0,93 & 4,19 & 0,99 & 3,84 & 1,16 & 4,20 & 0,97 \\
\hline Deportes electrónicos & 3,66 & 1,17 & 4,06 & 1,03 & 4,34 & 0,90 & 3,40 & 1,35 \\
\hline Ultímate & 4,16 & 1,08 & 3,57 & 1,33 & 3,68 & 1,21 & 4,11 & 1,03 \\
\hline Rugby subacuático & 4,08 & 1,09 & 4,15 & 1,14 & 4,00 & 1,11 & 3,97 & 1,05 \\
\hline Kayak & 3,86 & 1,17 & 3,91 & 1,29 & 3,88 & 1,15 & 3,78 & 1,09 \\
\hline Raftin & 3,88 & 1,14 & 3,70 & 1,38 & 3,77 & 1,06 & 3,71 & 1,06 \\
\hline Agility & 3,76 & 1,11 & 3,88 & 1,26 & 3,88 & 1,10 & 3,53 & 1,31 \\
\hline Lacrosse & 3,89 & 1,07 & 3,52 & 1,36 & 3,98 & 1,17 & 3,86 & 1,10 \\
\hline Roller derby & 3,99 & 1,01 & 3,78 & 1,14 & 3,77 & 1,18 & 3,85 & 1,09 \\
\hline Slackline & 3,95 & 1,03 & 3,81 & 1,16 & 3,80 & 1,10 & 3,74 & 1,09 \\
\hline Salto doble cuerda & 3,92 & 1,01 & 3,69 & 1,18 & 3,86 & 1,03 & 3,68 & 1,00 \\
\hline Skateboarding & 3,92 & 1,09 & 3,87 & 1,23 & 3,89 & 1,02 & 3,82 & 1,00 \\
\hline Downhill & 4,01 & 1,06 & 3,75 & 1,26 & 3,75 & 1,13 & 4,02 & 0,99 \\
\hline Escalada & 4,07 & 1,01 & 3,85 & 1,20 & 3,95 & 1,21 & 3,96 & 1,03 \\
\hline Senderismo & 4,01 & 1,04 & 3,93 & 1,16 & 3,98 & 1,15 & 3,87 & 1,18 \\
\hline Orientación & 3,85 & 1,02 & 3,82 & 1,30 & 3,86 & 1,21 & 3,72 & 1,19 \\
\hline Caminata campo traviesa & 3,96 & 1,03 & 3,57 & 1,28 & 3,96 & 1,08 & 3,88 & 1,03 \\
\hline Ciclomontañismo & 4,02 & 1,10 & 3,72 & 1,32 & 3,70 & 1,13 & 3,96 & 1,14 \\
\hline Freestyle & 4,01 & 1,02 & 3,58 & 1,44 & 3,89 & 1,20 & 3,95 & 1,10 \\
\hline Parkour & 4,09 & 0,99 & 3,73 & 1,26 & 4,18 & 0,99 & 3,97 & 1,12 \\
\hline BMX & 4,12 & 1,11 & 3,97 & 1,30 & 4,27 & 1,00 & 4,09 & 1,07 \\
\hline
\end{tabular}




\section{Inclusión de las Prácticas Emergentes}

La comunidad educativa ( $n=428$ ) consideró que las cinco actividades físicas que deben ser incluidas en el plan de estudios y que obtuvieron mayor promedio fueron en su orden: entrenamiento funcional $(4,46)$, deportes adaptados $(4,34)$, crossfit $(4,32)$, ultimate $(4,03)$ y ciclomontañismo $(3.86)$ (ver tabla 4$)$.

Tabla 4. Estadísticos descriptivos de inclusión de Prácticas Emergentes

\begin{tabular}{|l|c|c|}
\hline Variables & Media & Desv. típ. \\
\hline Entrenamiento funcional & 4,46 & 0,94 \\
\hline Deportes adaptados & 4,34 & 0,99 \\
\hline Crossfit (metodología) & 4,32 & 1,05 \\
\hline Ultímate & 4,03 & 1,19 \\
\hline Ciclomontañismo & 3,86 & 1,23 \\
\hline Street Workout & 3,76 & 1,26 \\
\hline Agility & 3,2 & 1,39 \\
\hline Slackline & 3,29 & 1,26 \\
\hline Salto doble cuerda & 3,33 & 1,28 \\
\hline Escalada & 3,55 & 1,27 \\
\hline Senderismo & 3,75 & 1,24 \\
\hline Orientación & 3,57 & 1,32 \\
\hline Caminata campo traviesa & 3,51 & 1,29 \\
\hline Freestyle & 3,51 & 1,29 \\
\hline Parkour & 3,57 & 1,31 \\
\hline
\end{tabular}

Los cuatro actores del currículo coinciden en la importancia de incluir e implementar un área flexible, electiva, que contenga las prácticas emergentes como lo son el entrenamiento funcional, los deportes adaptados como una modalidad deportiva que se adapta al colectivo de personas con discapacidad o condición especial de salud, el crosfitt mediante el cual se pueden trabajar simultáneamente, entre otras diez grandes áreas físicas: agilidad, coordinación, equilibrio, flexibilidad, fuerza, potencia, precisión, resistencia cardiorrespiratoria, resistencia muscular y velocidad. Así mismo se destaca el ultimate como un deporte de valores, que no requiere un juzgamiento externo, sino que son los mismos actores quienes median y finalmente impulsado por la geografía colombiana ha hecho del ciclomontañismo un deporte atractivo porque se practica al aire libre, en la naturaleza, generando hedonismo, vértigo, riesgo y placer. Al realizar el análisis de acuerdo al tipo de población (ver tabla 5), se encontró que la población de estudiantes $(4,56)$, empresarios $(4,46)$ y egresados $(4,55)$ presentan su mayor promedio en la inclusión del Entrenamiento Funcional en el plan de estudios; por su parte los docentes $(4,43)$ presentan como primera opción el Crossfit.

Tabla 5. Estadísticos descriptivos de inclusión de Prácticas Emergentes de acuerdo a la muestra

\begin{tabular}{|l|c|c|c|c|c|c|c|c|}
\hline & \multicolumn{2}{|c|}{$\begin{array}{c}\text { Estudiantes } \\
(\mathrm{n}=186)\end{array}$} & \multicolumn{2}{c|}{$\begin{array}{c}\text { Docentes } \\
(\mathrm{n}=67)\end{array}$} & \multicolumn{2}{c|}{ Empresarios $(\mathrm{n}=56)$} & \multicolumn{2}{c|}{$\begin{array}{c}\text { Egresados } \\
(\mathrm{n}=119)\end{array}$} \\
\hline Variables & Media & $\begin{array}{c}\text { Desv } \\
\text { típ. }\end{array}$ & Media & $\begin{array}{c}\text { Desv. } \\
\text { típ. }\end{array}$ & Media & $\begin{array}{c}\text { Desv. } \\
\text { típ. }\end{array}$ & Media & $\begin{array}{c}\text { Desv. } \\
\text { típ. }\end{array}$ \\
\hline Crossfit (metodología) & 4,36 & 1,02 & 4,43 & 0,84 & 4,20 & 1,17 & 4,26 & 1,14 \\
\hline Entrenamiento funcional & 4,56 & 0,89 & 4,06 & 1,14 & 4,46 & 0,95 & 4,55 & 0,83 \\
\hline Street Workout & 3,72 & 1,23 & 4,19 & 1,20 & 3,80 & 1,20 & 3,55 & 1,34 \\
\hline Deportes adaptados & 4,52 & 0,79 & 3,54 & 1,22 & 4,46 & 0,85 & 4,45 & 0,99 \\
\hline Ultímate & 4,08 & 1,20 & 4,19 & 1,06 & 3,91 & 1,21 & 3,91 & 1,22 \\
\hline Agility & 3,17 & 1,36 & 3,16 & 1,39 & 3,45 & 1,29 & 3,13 & 1,47 \\
\hline Slackline & 3,37 & 1,26 & 3,21 & 1,31 & 3,39 & 1,22 & 3,18 & 1,26 \\
\hline Salto doble cuerda & 3,38 & 1,32 & 3,42 & 1,22 & 3,39 & 1,20 & 3,18 & 1,28 \\
\hline Escalada & 3,64 & 1,27 & 3,40 & 1,29 & 3,64 & 1,26 & 3,46 & 1,27 \\
\hline Senderismo & 3,75 & 1,22 & 3,79 & 1,17 & 3,86 & 1,27 & 3,67 & 1,30 \\
\hline Orientación & 3,46 & 1,30 & 3,66 & 1,31 & 3,84 & 1,25 & 3,59 & 1,37 \\
\hline Caminata campo traviesa & 3,52 & 1,28 & 3,52 & 1,31 & 3,52 & 1,26 & 3,47 & 1,33 \\
\hline Ciclomontañismo & 3,91 & 1,28 & 3,84 & 1,15 & 3,82 & 1,16 & 3,79 & 1,25 \\
\hline Freestyle & 3,65 & 1,26 & 3,31 & 1,31 & 3,41 & 1,32 & 3,47 & 1,31 \\
\hline Parkour & 3,63 & 1,33 & 3,36 & 1,32 & 3,59 & 1,23 & 3,56 & 1,31 \\
\hline
\end{tabular}




\section{Datos cualitativos. Resultados del grupo focal}

Se aplicaron entrevistas semiestructuradas a un grupo focal conformado por ocho expertos del área de la actividad física, ejercicio físico, el deporte y la recreación; quienes representan y lideran los procesos relacionados con las prácticas emergentes en los entes deportivos. Las entrevistas estuvieron dirigidas a cinco personas del sector productivo, un graduado, un estudiante y un docente. Para los entrevistados la palabra común con la que se definieron las Prácticas emergentes fue "nuevas tendencias", "nuevos deportes", "nuevas modalidades", "nuevas alternativas", "nuevos mercados" y "alternativas diferentes". En su orden, las cinco Prácticas Emergentes que los integrantes del grupo focal consideran tienen mayor penetración en la ciudad de Medellín fueron: Skateboarding, Street workout, Roller freestyle y el BMX, adicionalmente agregan que deberían ser incluidas como asignaturas electivas en el plan de estudios del Profesional en Deporte, ya que permitiría a los graduados adquirir nuevas competencias y mayores oportunidades laborales. De acuerdo con lo anterior se evidencia una coincidencia parcial con las respuestas dadas por la comunidad educativa del programa.

La emergencia de estas nuevas tendencias, ha permitido la unificación de varias prácticas deportivas en una sola, logrando así, aprovechar el tiempo para destinarlo a otras actividades. Al respecto, Mármol et al. (2014) plantean que el crossfit es una práctica innovadora y emergente debido a sus características de entrenamiento y metodología, que brindan la posibilidad de integrar varias competencias básicas. El crossfit como práctica emergente viene creciendo y sobresaliendo como tendencia elegida por el sector productivo, estudiantes y docentes. Así mismo el entrenamiento funcional genera cada vez más adeptos dentro de los gimnasios, por tratarse de una práctica novedosa basada en ejercicios cortos de alta intensidad que generan un gran gasto calórico. Esto ha despertado el interés tanto de entrenadores y estudiantes como de usuarios activos de estos espacios de acondicionamiento físico, que ven en el entrenamiento funcional una manera de potenciar y sacar el mayor provecho de su entrenamiento rutinario. Los materiales empleados para el desarrollo de esta actividad física, son fáciles de transportar y pueden realizarse en cualquier parque o espacio al aire libre. Hoy no sólo se busca preservar la salud, también el mejoramiento de las condiciones físicas y la obtención de una apariencia ajustada a unos estereotipos sociales establecidos.

De acuerdo con lo anterior surge la importancia de tener en cuenta esas nuevas tendencias, donde lo emergente se manifiesta social y culturalmente; "la emergencia de nuevas tendencias de la cultura corporal (...) sedimentan otros patrimonios corporales en los sujetos que habitan las ciudades" (Cachorro et al., 2009, p. 44). Deportes como el Ultimate, Entrenamiento funcional, Crossfit, Slackline, los fitness el entrenamiento por aceleración y el fútbol femenino se encuentran dentro de esas actividades físicas y deportivas que han acogido a todo tipo de personas de diferentes edades y géneros. Pero se debe tener en cuenta algo muy importante y es que el actual modelo neoliberal arrastra al consumo y a la belleza como ejes fundamentales de estas nuevas actividades.

Lo que logra inferir de los análisis estadísticos y las entrevistas, es que en la actividad física y el deporte, afloran nuevas realidades de relación social, cultural y ambiental, representadas por prácticas emergentes que dan lugar a nuevos fundamentos o fuentes del currículo que se constituyen en "posiciones de índole socio-cultural, epistemológica-profesional y psicopedagógica, a través de las cuales se derivan principios que deberían orientar tanto el diseño curricular como su desarrollo y evaluación" (Casarini, 2012, p.39). Las posiciones o supuestos, "resultantes de la particular visión de dichos fundamentos", permite responder a las preguntas ¿Por qué y para qué enseñar-aprender? ¿Qué enseñar-aprender? ¿Cuándo enseñar-aprender? ¿Cómo enseñar-aprender? ¿Qué, cuándo y cómo evaluar?

\section{DISCUSIÓN}

Los resultados indican que en el contexto de la ciudad de Medellín las cinco prácticas emergentes con mayor acogida y en su orden fueron: Crossfit, entrenamiento funcional, deportes adaptados, entrenamiento en suspensión y Street Workout. En relación con el Crossfit la Encuesta nacional de tendencias de fitness en España (Veiga et al., 2017) la ubico en el puesto 17 y para el año 2020 esta tendencia no figura en este ranking (Cámara et al., 2020), claro está, que los autores lo mencionan como un entrenamiento de fuerza utilizado frecuentemente en los centros de fitness como entrenamiento básico en la rutina, ya que es base de este tipo entrenamiento y es muy utilizado en la actualidad.

Otra práctica que emergió con fuerza en el presente estudio fue el entrenamiento funcional; que en comparación con el estudio de Veiga et al. (2017) se situó en el tercer lugar; y para la misma encuesta para el 2020 bajó dos lugares quedando en el quinto lugar en relación con el 2019, "aunque se mantiene dentro de las 5 primeras posiciones, como en años anteriores y se sitúa por encima de la posición que alcanzó en la encuesta internacional (puesto 7)" (de la Cámara et al., 2020, p. 438) 
En comparación con el China fitness trends for 2020 (Li et al., 2019) se coincide únicamente con el entrenamiento funcional, el cual se colocó en el cuarto lugar, tendencia que según el autor busca mantener y aumentar la fuerza muscular y fortalecer el físico mediante el uso de peso corporal, peso libre o máquinas. En China el entrenamiento funcional se clasificó como numero 1 tanto en 2018 como en 2019". Li et al. (2019) sostienen que "aunque la encuesta global eliminó esta tendencia potencial en 2019 porque se determinó que era demasiado genérica, permaneció en la encuesta de China" (p. 22).

En cuanto a los deportes adaptados, se ubicaron en el tercer puesto en la presente investigación en comparación con el estudio de Cámara et al. (2020) que aparecen con el nombre de "Prevención/readaptación funcional de lesiones", y que ocupa el onceavo puesto y se ha convertido en una tendencia que ha ido obteniendo buena acogida como se evidencia en la encuesta española en 2018, ya que ha ido mejorando su posición. "Esta tendencia ha pasado de estar en los últimos puestos del top 20 de 2018 y 2019 a estar cerca del top 10, por lo que será interesante si se consolida como tendencia en el sector del fitness español" (Cámara et al., 2020) p. 439). En cuarto lugar se encontró el entrenamiento en suspensión, el cual no figura en Veiga et al. (2017), ni en el listado de las Tendencias Encuesta Nacional 2020 (De la Cámara, 2020). En el quinto lugar está el Street Workout que tampoco figura en estos dos ranking españoles. Esto mismo sucede para las otras prácticas valoradas por el grupo focal: Skateboarding, Street workout, Roller freestyle y el BMX.

De las tendencias encontradas en esta investigación ninguna coincide con el "Top 20 Worldwide Fitness Trends for 2019" (Thompson, 2018), cuyos primeros cinco puestos fueron ocupados por: Wearable technology, Grouptraining, High-intensity interval training (HIIT), Fitness programs for older adults $y$ Bodyweight training. De igual manera, no se encontró coincidencia con el "Top 20 Worldwide Fitness Trends for 2018" (Thompson, 2017) cuyos primeros lugares los ocuparon: High-intensity interval training, Group training, Wearable technology, Body weight training, y Strength training. Las tendencias encontradas para Medellín, solamente coincide con una del "Top 20 China Fitness Trends for 2020, la tendencia del "Strength training" (p. 24). Las primeras de ese top fueron en su orden: exercise for weight loss, exercise and sport for children and adolescents; Exercise is Medicine; Strength training y Fitness and dietary; la segunda, cuarta y quinta específicos para China.

En este sentido, el "Strength training" figuraba en el "Top 20 Worldwide Fitness Trends for 2017" (Thompson, 2016). Para ese año las primeras tendencias fueron: wearable technology, body weight training, high-intensity interval training, educated, certified, and experienced fitness professionals y strength training (p. 11). El comparativo de estos resultados con "Top 20 Worldwide Fitness Trends for 2016" en el Worldwide survey of fitness trends for 2016 (Thompson, 2015, p. 12) son similares, y para este año el strength training ocupaba el puesto cuarto; las cinco mejores posicionadas en este top fueron: wearable technology, body weight training, high-intensity interval training (HIIT), strength training $y$ educated, certified, and experienced fitness professionals.

Esto muestra una gran diferencia de tendencias e intereses. Para China, los resultados de la encuesta de aptitud física para 2020, junto con los de 2018 y 2019, indican que había seis tendencias específicas en este país clasificadas entre las 20 principales para 2020, que fueron recogidas de las 12 tendencias potenciales nominadas por expertos chinos en salud y fitness; señalando algunas tendencias mundiales como el entrenamiento en intervalos de alta intensidad. Específicamente para este país, las aplicaciones móviles de ejercicio, la tecnología portátil, la intervención para niños y adolescentes con sobrepeso y obesidad, y la caminata rápida volvieron al top 20 después de haber salido del top 20 el año pasado. Lo anterior indica la importancia de las especificaciones de cada país de las tendencias para poderlos incluir en planes y programas educativos, lo cual coincide con Li et al. (2019) cuando dicen que "las organizaciones o empresas internacionales de fitness deben considerar la especificidad nacional de las tendencias de fitness al realizar planes estratégicos en otros países" (p. 26)

En relación con estas tendencias globales del fitness y en contraposición con los deportes formales de ligas y clubes, en el contexto de la enseñanza aparece un estudio recién publicado de la Universidad de Monash en Australia de O'Connor y Penney (2020) quienes sostienen que internacionalmente, los patrones de participación en el deporte están cambiando, y la denominada participación "informal" desplaza la participación en el deporte basada en clubes y otras actividades estructuradas formalmente, así la educación física puede alinearse pedagógicamente con las tendencias de participación contemporáneas, para ayudar a crecer y mantener la participación de los jóvenes en el deporte más allá de las escuelas.

El surgimiento en Medellín de prácticas emergentes que no están incluidas en el top de las encuestas globales, indica que cada lugar tiene especificidades y tendencias locales que predominan por encima de la tendencia global, como puede verse con la práctica del Street Workout, que aparece tanto en el consolidado como en el grupo focal, así como el Skateboarding, Roller freestyle y el BMX consideradas tendencias en Medellín, incidiendo en las tendencias culturales y deportivas de la ciudad. 
En este sentido, las prácticas corporales poseen diferentes niveles de transformación, ruptura e innovación que combinan elementos que generan una dirección incierta, por lo que en estas prácticas se pueden vislumbrar las prevalencias y predominios de las disposiciones de ser y postura frente a la vida y de este modo, los modelos que procuran la conservación. "La regularidad es el círculo vicioso de algunos practicantes que no se apartan de una preferencia corporal, por lo cual no se asumen como meras reproducciones de ideas lineales" (Cachorro, 20098). Estos elementos se ratifica en Zaragoza (2017) con el Padbol, deporte alternativo que como practica corporal no convencional no está incluido en los espacios curriculares de la Educación Física, esta falta de oficialidad y de consenso global se manifiesta en que "no posee una decodificación sustentable de lenguaje específico y no fue foco de investigaciones como los deportes reconocidos, pero que el padbol no pertenezca a dicho entorno no imposibilita que no pueda ser practicado por el común de la sociedad" .

La inclusión de las prácticas emergentes o prácticas corporales en el currículo es sustentado con estudios validados como los de Chiva et al. (2016) que incluyen la práctica del Aps, una propuesta pedagógica más allá de las aulas que combina aprendizaje y servicio comunitario, basada en la implementación de habilidades relacionados con los contenidos curriculares realizados en contextos reales; Es decir, y como lo plantean Palacio et al. (2019) los objetivos y contenidos de las asignaturas deben establecer una relación explicita con los perfiles de egreso de los estudiantes y no por el contrario, una distancia entre el proceso de formación universitaria y el quehacer profesional, tal como lo encontraron Icarte y Labate (2016).

En la revisión de los planes de estudio de otros programas profesionales en actividad física y deporte en Medellín, se encontró que los resultados de esta investigación no están presentes en sus currículos; por ejemplo en el programa de entrenamiento deportivo de la Universidad de Antioquia, los estudiantes pueden seleccionar un deporte en específico, incluidos los deportes paralímpicos, pero no se menciona en ningún momento las prácticas emergentes o nuevas tendencias. Así mismo, en el programa de Actividad Física y Deporte de la Universidad Católica Luis Amigó, se expone la asignatura "Expresiones motrices emergentes: tendencias en fitness y wellness" enfocado a la asesoría e intervención en procesos de entrenamiento personalizado, sesiones grupales, gimnasios y otros escenarios; es necesario mencionar que para el presente estudio, el wellness no hizo parte de las resultados encontrados. Finalmente en el programa de entrenamiento deportivo del Servicio Nacional del Aprendizaje (Sena) se tienen en cuenta el entrenamiento deportivo y el conocimiento de los procesos y las tendencias de las modalidades deportivas.

Es importante señalar que las prácticas emergentes pueden ser tendencias globales que van siendo populares entre las poblaciones de los diferentes países y que se van convirtiendo en tendencia global en contraposición con las prácticas deportivas formales como los deportes de clubes y ligas deportivas. Existe una contraposición entre estas dos prácticas, lo que hace que el currículo se debe cuestionar en la actualidad sobre la importancia de actualizar sus métodos de enseñanza y sus contenidos para que estén más acordes a las tendencias globales.

\section{CONCLUSIONES}

A partir de los resultados obtenidos, se pueden extraer las siguientes conclusiones: 1) El currículum universitario del programa profesional en deporte necesita generar en los estudiantes habilidades para su desempeño profesional, siendo necesario abordar el conocimiento de las Practicas Emergentes, ya que es una tarea urgente para ofrecer herramientas necesarias y actuales que den respuesta a las transformaciones sociales y culturales; 2) La inclusión de asignaturas electivas tales como: CrossFit, Skateboarding, deportes adaptados, Street workout y Ultimate debe acogerse como una oportunidad y no como una amenaza en el proceso de formación profesional de los estudiantes del campo del deporte; 3) Las tendencias encontradas para Medellín coinciden muy poco con las actuales tendencias globales, esto porque se están generando dentro de la ciudad unas prácticas corporales que buscan salirse de los patrones establecidos, lo que va generando a la vez, identidad local a las prácticas emergentes.

\section{REFERENCIAS}

Águila, C., y Sicilia, Á., La actividad física en España ante la dialéctica modernidad-posmodernidad: el caso de los centros fitness, Movimento, 20 (3), 1223-1242 (2014)

Cachorro, G.A., Prácticas corporales. Traducción de sentidos en la ciudad, Pensar a Prática, 12(2), 1-10 (2009)

Chiva, Ò., Gómez, J.G., Martínez, R.C., y Peris, C.C., El aprendizaje servicio como propuesta metodológica para una pedagogía crítica, Ridas Revista Iberoamericana de Aprendizaje Servicio, 2, 70-94 (2016)

De la Cámara, M.A., Torrente, M.V., y Veiga, Ó.L., Encuesta nacional de tendencias de fitness en España para el año 2020, Retos Nuevas Tendencias en Educación Física, Deporte y Recreación, 37, 434-441 (2020)

García, F.M., y Llopis G.R., Encuesta sobre los hábitos deportivos en España 2010, $1^{\text {a }}$ Ed., 1-270, Ideal Democrático y Bienestar Personal, Madrid, España (2011) 
Gaviria, N.A., Pulido, S.M., y otros tres autores, Prácticas deportivas emergentes en Medellín pura adrenalina, $1^{\mathrm{a}}$ Ed., 1 130, INDER Alcaldía de Medellín, Medellín, Colombia (2015)

Garganta, J., Trends of tactical performance analysis in team sports: bridging the gap between research, training and competition, Revista Portuguesa de Ciências do Desporto, 9(1), 81-89 (2009)

Grisales H., Muestreo en estudios descriptivos, Medellín, Facultad Nacional de Salud Pública-Universidad de Antioquia, (2000)

Icarte, G.A., y Labate, H.A., Metodología para la Revisión y Actualización de un Diseño Curricular de una Carrera Universitaria Incorporando Conceptos de Aprendizaje Basado en Competencias, Formación Universitaria, 9(2), 3-16 (2016)

Kumar, R., Innovations in sports and physical education classes, International Journal of Physical Education, Sports and Health, 4(1), 273-276 (2017)

Li, Y.M., Han, J., y otros cinco autores, China survey of fitness trends for 2020, ACSM's Health \& Fitness Journal, 23(6), 19-27 (2019)

López, M., Prácticas y fenómenos emergentes en la juventud como vías de transformación social en Colombia, Última Década, 35, 33-59 (2011)

Mata, D., Deporte: cultura y contracultura. Un estudio a través del modelo de los horizontes deportivos culturales, Apunts Educación Física y Deportes, 1(67), 6-16, (2002)

Mármol, A., Sánchez, B., Pérez, M., y Ribes, A., El crossfit en la educación física escolar, EmásF Revista Digital de Educación Física, 30, 19-34 (2014)

OECD, Future of education 2030 making physical Education dynamic and inclusive for 2030, 1, 1-106, Monkey Business Images, Paris, France (2019)

O'Connor, J., y Penney, D., Informal sport and curriculum futures: An investigation of the knowledge, skills and understandings for participation and the possibilities for physical education, European Physical Education Review, 27(1), 3-26 (2020)

Palacio, J.A., Hinojo, F.J., y Rodríguez, A.M., Desarrollismo pedagógico en el programa de Tecnología Agropecuaria del Politécnico Colombiano Jaime Isaza Cadavid, Formación Universitaria, 12(5), 79-90 (2019)

Polit, D., y Hungler, B., Investigación científica en ciencias de la salud: principios y métodos. 6ª ed., McGraw-Hill, Book Co., México (2005)

Sánchez, R., y Sánchez, J., Culturas deportivas y valores sociales: una aproximación a la dimensión social del deporte. Apunts. Educación Física y Deportes, 2 (64), 33-45 (2001)

Rath, S.S., Current trends of physical education and its future prospects in relation to individual's health, International Journal of Yogic, Human Movement and Sports Sciences, 2(1), 01-03 (2017)

Thompson, W.R., Worldwide survey of fitness trends for 2020, ACSM's Health \& Fitness Journal, 23 (6) 10-18 (2019)

Thompson, W.R., Worldwide survey of fitness trends for 2019. ACSM's Health \& Fitness Journal, 22(6), 10-17 (2018)

Thompson, W.R., Worldwide survey of fitness trends for 2018: the CREP edition, ACSM's Health \& Fitness Journal, 21(6), 10-19 (2017)

Thompson, W.R., Worldwide survey of fitness trends for 2016: 10th anniversary edition, ACSM's Health \& Fitness Journal, 19(6), 9-18 (2015)

Veiga, O.L., Valcarce., T.M. y King, C.A., National survey of fitness trends in Spain for 2017, Apunts Educación Física y Deportes, 128 (2), 108-125 (2017)

Zaragoza, J.N., Prácticas corporales no convencionales y deportes alternativos emergentes: geografía, territorio y espacios humanos, XII Congreso Argentino y VII Latinoamericano de Educación Física y Ciencias, Ensenada, 1-11 (2017) 